\title{
Copenhagen no more
}

\section{The opportunity of reaching a strong agreement on carbon emission cuts must not be missed again.}

It was anticipated as the fundamental meeting to define a global strategy on how to tackle climate change. It should have been the venue for the completion of a deal on carbon emissions cuts for individual countries. Instead, in spite of a long preparation and after lengthy and argumentative discussions, the United Nations Climate Change Conference in Copenhagen ended with a highly disappointing result.

In short, it was agreed that efforts should be made to keep temperature rises within $2{ }^{\circ} \mathrm{C}$ from pre-industrial levels, which the scientific community has long considered the threshold for dangerous climate change effects. That a legally binding deal was unlikely to be reached had been clear for weeks, but there was hope for a political agreement on carbon emission cuts by 2020 and 2050. But even this was not achieved. What was agreed was an investment of $\$ 30$ billion by 2012 and up to $\$ 100$ billion a year in 2020 from developed countries to support the growth of clean technologies in the developing world. But this will not lead to climate change mitigation.

The Fourth Assessment Report of the Intergovernmental Panel on Climate Change (IPCC) (http://go.nature. com/1Q7Vp5), published in 2007, concluded that the observed rise in temperature since pre-industrial values was most certainly caused by greenhouse gas emissions - primarily $\mathrm{CO}_{2}$ - owing to human activity. Continuing emissions may lead to devastating effects on the climate, unless they peak before 2020 and reduce rapidly afterwards. The results of climate research since 2007 were reviewed in The Copenhagen Diagnosis, published last December by a number of climate scientists (http://www.copenhagendiagnosis.org/). Not only do these results confirm the conclusions of the fourth IPCC report, but they make the picture even gloomier.

In light of such wide scientific consensus that human activities, particularly in the developed world, may lead to irreversible consequences for life on the planet, we should all feel a responsibility to cut carbon emissions. Unfortunately, awareness of the scientific evidence does not directly translate into concrete action.

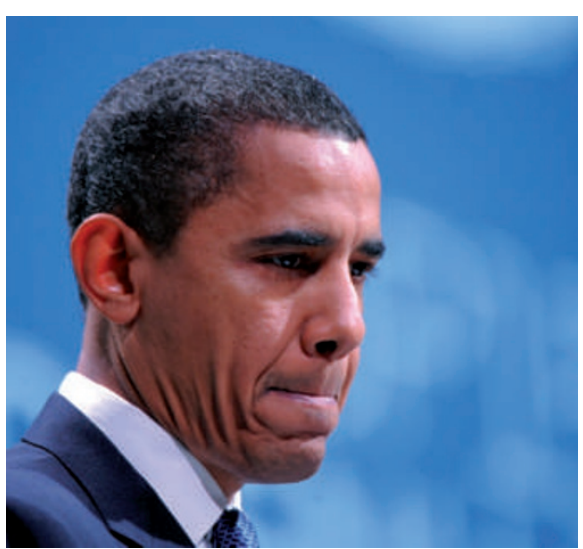

To put it simply, cutting emissions is too expensive. Fossil fuels, the primary source of $\mathrm{CO}_{2}$, are still far cheaper than any existing carbon-free energy source, and governments are reluctant to take drastic measure that could damage their economies. Public awareness has certainly increased, but this is not enough to make a difference. Temperature rises will seriously affect most parts of the developed world in a few decades, and ordinary citizens are unlikely to voluntarily pay more for carbon-free energy, or simply to give up a comfortable lifestyle to which they have been used to for a long time. Any matter with immediate effect, like the recent economic crisis, will naturally take priority in the public's opinion.

\section{In light of such wide}

scientific consensus that

human activities may lead to irreversible consequences for life on the planet, we should all feel a responsibility to cut

\section{carbon emissions.}

A legally binding deal that forces countries to cut their emissions is therefore our best bet for success. It would oblige governments to artificially increase the costs of emissions, resulting in higher investments in research and development of alternative energy sources, as well as in a natural reduction of energy consumption by industry and the general public.
An effective deal has essential requirements: it should establish clear penalties for countries not meeting their targets, which is one of the main criticisms made of the Kyoto Protocol. Agreeing on such penalties is not simple. For example, the possibility of trade sanctions, supported by France, may work, but is not seen positively by most countries.

Surely a deal should include the US and China, which in equal parts account for about $40 \%$ of all emissions. Indeed, the lack of commitment from these two countries is considered by many as the principal reason for failure in Copenhagen. It certainly made pointless the good intentions of others, like the European Union's willingness to increase its target for 2020 from 20\% to $30 \%$ cuts compared with 1990 levels. President Obama's attitude to climate change is radically different from that of his predecessor, and his intention to cut emissions by $4 \%$ with respect to 1990 by 2020, announced a few weeks before the meeting, was a positive sign. But imposing emission cuts on a country with an economy largely based on fossil fuels is no easy task, as shown by the difficulty he is encountering in getting the Clean Energy and Security Act approved. Surely, the lack of more decisive commitments in Copenhagen has widely disappointed those expecting strong leadership at an international level. Regarding China, despite the fact that $80 \%$ of the country's energy comes from coal, clear signs for investments in clean technology have been seen in recent legislation. But the refusal to be subjected to emissions monitoring has certainly not helped in reaching a more substantial agreement.

The failure to reach even a political agreement will mean further delays for a legally binding one. Many agree that the deadline for such a goal should be the next UN conference in Mexico, at the end of 2010. Should that really be achieved, a single year delay would probably not have a huge effect on the climate in decades from now. But every year of delay will increase the chances of irreversible global warming. The planet and its inhabitants simply cannot afford any more missed opportunities. 